fluency (effect size $d=0.9, P=0.071$ ), compared with the late remitters.

Our group has just completed the Mood Disorders in Elderly treated with Convulsive Therapy (MODECT) study, which included 110 patients with a mean age of 73 years (range 55-90 years). This study aims to identify predictors for the efficacy of ECT using neuroimaging, clinical measures (on cognition, mood and psychomotor symptoms), neuropsychological data and biological measurements. Recently, another research group in The Netherlands presented exciting data using a functional magnetic resonance imaging marker for the prediction of individual ECT outcome. ${ }^{2}$ The MODECT data provide a wonderful opportunity to study and possibly replicate these findings in an older cohort.

With respect to the optimal treatment modality, we agree that the speed of remission using ultra-brief pulse ECT in the PRIDE study was indeed comparable to the speed of remission of the merged ultra-brief/brief pulse ECT groups. ${ }^{3}$ However, the assessments of week 2 of the ECT group were neglected for comparison with the medication group. In the original ECT study, ${ }^{4}$ this elderly, brief pulse subgroup achieved remission significantly faster than the elderly, ultra-brief pulse subgroup: remission was achieved in 2.2 weeks (s.d. $=0.9)$ v. 3.0 weeks (s.d. $=1.1 ; t(29)=-2.249$, $P=0.032$ ), respectively. This finding may denote the possibility that twice-weekly brief pulse ECT with either unilateral or bilateral electrode placement could have superior efficacy compared with ultra-brief pulse treatment.

The recent evidence shown by our research and the recent findings of the PRIDE study once more emphasise the clinical importance of ECT's rapid effect; ECT should indeed be taken into account when revising treatment algorithms for severely depressed elderly patients, hence avoiding the use of the less effective and slower-acting antidepressant medication.

1 Rhebergen D, Huisman A, Bouckaert F, Kho KH, Kok RM, Sienaert P, et al. Older age is associated with rapid remission of depression after electroconvulsive therapy: a latent class growth analysis. Am J Geriatr Psychiatry 2014; doi: 10.1016/j.jagp.2014.05.002.

2 van Waarde JA, Scholte HS, van Oudheusden $U$, Verwey $B$, Denys $D$, van Wingen GA. A functional MRI marker may predict the outcome of electroconvulsive therapy in severe and treatment-resistant depression. Molecular Psychiatry 2014; doi: 10.1038/mp.2014.68.

3 Spaans HP, Sienaert P, Bouckaert F, van den Berg JF, verwijk E, Kho KH, et al. Speed of remission in elderly patients with depression: electroconvulsive therapy v. medication. Br J Psychiatry 2015; 206: 67-71.

4 Spaans HP, Verwijk E, Comijs HC, Kok RM, Sienaert P, Bouckaert F, et al Efficacy and cognitive side effects after brief pulse and ultrabrief pulse right unilateral electroconvulsive therapy for major depression: a randomized, double-blind, controlled study. J Clin Psychiatry 2013; 74: e1029-36.

\footnotetext{
The ResPECT (Research in Psychiatry and ECT) group: Harm-Pieter Spaans, Parnassia Psychiatric Institute, The Hague, The Netherlands. Email:

hp.spaans@parnassia.nl. Pascal Sienaert, Professor, Department of Psychiatry, University Psychiatric Centre - KU Leuven, campus Kortenberg, Belgium,

Filip Bouckaert, University Psychiatric Centre - KU Leuven, campus Kortenberg,

Belgium, Julia F. van den Berg, Parnassia Psychiatric Institute, the Hague,

The Netherlands, Esmée Verwijk, Parnassia Psychiatric Institute, the Hague,

The Netherlands, King H. Kho, Parnassia Psychiatric Institute, the Hague, The

Netherlands, Max L. Stek, Professor, Department of Elderly Psychiatry, VU University

Medical Centre and GGZ in Geest, Amsterdam, The Netherlands, Rob M. Kok

Parnassia Psychiatric Institute, the Hague, The Netherlands.

doi: $10.1192 /$ bjp.206.2.167a
}

\section{The 'unknown' safety concern for aripiprazole once monthly}

Fleischhacker et al report that treatment-emergent adverse effects are comparable for aripiprazole $400 \mathrm{mg}$ once monthly and a suboptimal dose $(50 \mathrm{mg})$ of aripiprazole once monthly. ${ }^{1}$ Also, they state that the 'clinical relevance' of statistically significant difference in Barnes Akathisia Rating Scale score with aripiprazole $400 \mathrm{mg}$ once monthly against oral aripiprazole is 'unknown'. Akathisia is known to be the most clinically relevant adverse effect with oral aripiprazole because of the subjective distress caused to the patient and the increased risk of agitation and suicide associated with it. Hence, a higher rate of akathisia with aripiprazole $400 \mathrm{mg}$ once monthly cannot be discounted as being of 'unknown clinical relevance'. Further, a deeper look at the apparently similar rates of 'any treatment-emergent adverse effects' for the two doses of aripiprazole reveals that the rates may not be similar if psychotic disorder and schizophrenia (which are efficacy outcomes and in no way can be considered as adverse effects for the purposes of this study) are removed from the list. The article minimises the possible safety concerns associated with aripiprazole $400 \mathrm{mg}$ once monthly. A precise assessment of safety concerns (besides efficacy) is of utmost importance for a potential prescriber and there is potential of a prescriber being misguided by superficially reading this article. Further, efficacy outcomes of the study could have been contaminated by the noticeably high and differential discontinuation rates in the two active arms. The last observation carried forward (LOCF) method used for analysis of missing data tends to underestimate worsening in intention-to-treat (ITT) analyses. A comparison of results generated by ITT and per protocol analysis could have been more informative in assessing the efficacy outcomes.

1 Fleischhacker wW, Sanchez R, Perry PP, Jin N, Peters-Strickland T, Johnson BR, et al. Aripiprazole once-monthly for treatment of schizophrenia: a double-blind, randomised, non-inferiority study. Br J Psychiatry 2014; 205 $135-44$

Sumit K. Gupta, Assistant Professor of Psychiatry, Abhilove Kamboj, Junio Resident (MD Course), Institute of Human Behaviour and Allied Sciences, Delhi, India Email: drsumit@aol.in

doi: 10.1192/bjp.206.2.168

Authors' reply: Gupta \& Kamboj correctly note that akathisia is a clinically relevant adverse effect with oral aripiprazole because it causes distress and is associated with an increased risk of agitation and suicide in patients with schizophrenia. We did not want to discount a higher rate of akathisia with aripiprazole $400 \mathrm{mg}$ once monthly as being of 'unknown clinical relevance', but rather questioned the clinical relevance of the absolute 0.11-point group difference on the 5-point Barnes Akathisia Global Scale. We appreciate that this could have been stated more clearly. In our study, ${ }^{1} 10.6 \%$ of patients treated with aripiprazole $400 \mathrm{mg}$ once monthly reported akathisia as a treatment-emergent adverse event (TEAE), as did $6.8 \%$ of patients treated with oral aripiprazole and $8.4 \%$ of patients treated with a sub-therapeutic dose of aripiprazole once monthly; no patients discontinued because of akathisia. Rates of agitation, reported as a TEAE, were low among all treatment groups (aripiprazole $400 \mathrm{mg}$ : 2.6\%; oral aripiprazole: $0.8 \%$; aripiprazole $50 \mathrm{mg}: 0 \%$ ). As noted in our manuscript, Clinical Global Impression Severity of Suicide (CGI-SS) scores and Columbia Suicide Severity Rating Scale (C-SSRS) suicidal ideation intensity total scores remained stable across treatment groups (see Table 4 in the published article ${ }^{1}$ ).

Gupta \& Kamboj note that the rate of TEAEs with aripiprazole $400 \mathrm{mg}$ once monthly may not be similar to the rate with a sub-therapeutic dose of aripiprazole once monthly if psychotic disorder and schizophrenia are removed from the list of TEAEs. They also suggested that psychotic disorder and schizophrenia are not TEAEs and are efficacy outcomes. In this context, we note that the regulatory authorities in Europe and the USA require accurate 